\title{
Cervical Spine Decompression and Fusion Outcomes in Trauma Patients Actively Receiving Anticoagulation Treatment for Cerebrovascular Injury: A Retrospective Comparative Study
}

\author{
FRANCIS X. CAMILLO, MD, ${ }^{1}$ SEAN M. MITCHELL, MD ${ }^{2}$

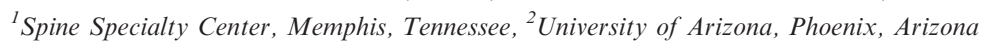

\begin{abstract}
Background: Cervical spine blunt trauma patients with the presence of a cerebrovascular injury may be given initiation of heparin anticoagulation treatment prior to necessary surgical stabilization. Literature regarding the safety and efficacy of these procedures while a patient is on active anticoagulation is limited, requiring further investigation. The primary research question for this study is: Can cervical spine decompression and fusion in the context of a blunt cerebrovascular injury and anticoagulation therapy be completed safely? To accomplish this a comparison of outcomes and perioperative complications was made to a control group.

Methods: A total of 63 trauma patients requiring cervical spine decompression and fusion from 2013 to 2015 were identified at our North American level 1 trauma center. Evaluation of patient injury data, bleeding events, postoperative infections, and neurologic outcomes was collected from chart review. The American Spinal Injury Association (ASIA) grading system was used to measure change in postoperative neurologic outcomes.

Results: Of 63 patients, 14 had a concomitant cerebrovascular injury that required perioperative anticoagulation treatment. In the 14 patients receiving anticoagulation, 11 had anterior and 3 had posterior stabilization. A total of 2 patients experienced a complication (pneumonia and hardware failure), but neither was related to anticoagulation. An elevated prothrombin time value was noted postoperatively in 1 patient, but with no adverse outcome. No bleeding or thrombotic events, surgical site infection, or neurologic deterioration occurred. The difference in postoperative ASIA grades between groups was not significantly different $(P=.57)$.

Conclusions: The operative cohort receiving anticoagulation therapy did not demonstrate an increase affinity for perioperative complications or a decline in ASIA scores postoperatively when compared to a control cohort.

Clinical Relevance: Patients with a cerebrovascular injury receiving anticoagulation treatment can undergo safe and successful cervical spine stabilization procedures.

Level of Evidence: Therapeutic level III.
\end{abstract}

Cervical Spine

Keywords: cervical spine trauma, decompression and fusion, cerebrovascular injury, complications, anticoagulation, heparin

\section{INTRODUCTION}

Cerebrovascular injuries occur more frequently with blunt trauma to the cervical spine than previously believed $(27.5 \%))^{1-3}$ If a cerebrovascular injury is left untreated, stroke and mortality rates in these patients can be as high as $50 \% .^{4,5}$ As a result, growing treatment trends have patients who present with cervical spine trauma screened for cerebrovascular injury before surgical stabilization of the spine so that treatment can be started early. ${ }^{2,6,7}$ Cerebrovascular injury can lead to neurovascular complications either from thrombus formation at the tear site or narrowing of the vessel. ${ }^{8}$ Anticoagulation treatment begun in the early period (before the onset of stroke symptoms) has been shown to improve mortality and lower neurologic complications, but in patients having spinal surgery it carries the risk of epidural hematoma and major bleeding. ${ }^{8-11}$ Although some studies provide recommendations as to when spine surgery patients should receive prophylaxis postoperatively to prevent deep vein thrombosis,, $12-14$ few directly discuss the outcomes in patients who are actively receiving anticoagulation treat- 
ment. ${ }^{15,16}$ Ideally anticoagulation treatment should be withheld until after spinal surgery has been completed, initiating treatment in the postoperative period. However, in the context of a cervical spine injury and a cerebrovascular injury, it is necessary to weigh the risks of a major bleed $(0.0 \%-4.3 \%)$ or epidural hematoma formation $(<1 \%)^{16}$ against the likelihood of a thrombotic or occlusive event. This is especially important if surgery has to be delayed beyond 12 hours. At our hospital all patients with blunt cervical spine injury are screened for cerebrovascular injury and therapy started according to institutional protocol.

The aim of this retrospective study was to assess outcomes and perioperative complications in trauma patients requiring cervical spine decompression and fusion in the context of a blunt cerebrovascular injury and anticoagulation therapy. This study sought to answer whether cervical spine stabilization can be performed successfully and safely in patients actively receiving anticoagulation treatment for a cerebrovascular injury. In order to accomplish this a comparison was made to a similar operative cohort of cervical spine trauma patients who did not have a cerebrovascular injury and did not receive anticoagulation treatment. This review did not explore the treatment of vertebral or carotid artery injuries; rather, the focus was on the outcomes of treatment and complications of the cervical spine surgery.

\section{MATERIALS AND METHODS}

After appropriate Institutional Review Board approval, 63 patients who met the inclusion criteria of cervical spine trauma (ligamentous disruption, loss of function, or cervical instability) that required a surgical stabilization procedure between May 2013 and November 2015 were identified at our North American level 1 trauma center. These patients represent the surgeries performed by 1 orthopaedic spine surgeon. The Institutional Review Board at this institution does not require patient informed consent for retrospective chart reviews with de-identified patient data. Data collected included the type and location of injury, the surgical procedure, the presence and location of cerebrovascular injury, preoperative and postoperative anticoagulation use, bleeding events, thrombosis, surgical site infections, neurologic injuries, complications, and time to fusion. The American
Spinal Injury Association (ASIA) grades before and after surgery were documented. In the ASIA scoring system, A represents a complete injury with no sensory or motor function; B represents incomplete injury with sensory function preserved but no motor function; $\mathrm{C}$ represents an incomplete injury with motor function preserved but a key muscle grade of less than 3; D represents an incomplete injury with motor function preserved and a muscle grade greater or equal to 3 ; and $\mathrm{E}$ represents normal.

All patients were screened for cerebrovascular injury at the time of presentation, and anticoagulation treatment was started when necessary according to the following protocol developed by the departments of trauma surgery, neurosurgery, and orthopaedic spine surgery at our institution.

- Patients presenting with any cervical trauma (including bone or soft tissue) meet the criteria for cerebrovascular injury screening with computed tomography (CT) angiography imaging to evaluate the arteries in the neck.

- Upon suspicion of vascular damage on initial CT angiography, further cerebral angiogram is completed to confirm cerebrovascular injury.

- After a cerebrovascular injury is diagnosed, an anticoagulation protocol is initiated, beginning a patient on a continuous low-intensity intravenous infusion of $15 \mathrm{U} / \mathrm{kg} / \mathrm{hr}$ unfractionated heparin. The partial thromboplastin time (PTT) range while on heparin is held between 40 and 60 seconds.

- The heparin drip is held 2 hours before any operative procedures, and it is resumed 4 hours postoperatively.

- Anticoagulation treatment was converted to aspirin $325 \mathrm{mg}$ and/or clopidogrel $75 \mathrm{mg}$ per day orally postoperatively as determined by the vascular service.

All patients underwent decompression and instrumented fusion (44 anterior and 19 posterior) by a single fellowship-trained orthopaedic spine surgeon. Proper hemostasis was achieved before wound closure in all patients, with drains placed at the end of surgery. Outputs were monitored by the orthopaedic operative service. Drains were pulled between 24 and 48 hours in each patient when an output of less than $50 \mathrm{cc}$ during a 12-hour shift was 
Table 1. Vertebral and cerebrovascular injury sustained, anticoagulation therapy given, stabilization received, and American Spinal Injury Association (ASIA) exam grade for each participant in our cerebrovascular injury patient cohort who received anticoagulation therapy perioperatively.

\begin{tabular}{|c|c|c|c|c|c|}
\hline Vertebral Injury & $\begin{array}{l}\text { Cerebrovascular } \\
\text { Injury }\end{array}$ & $\begin{array}{c}\text { Preoperative } \\
\text { Anticoagulation }\end{array}$ & $\begin{array}{l}\text { Postoperative } \\
\text { Anticoagulation }\end{array}$ & $\begin{array}{l}\text { Surgical } \\
\text { Procedure }\end{array}$ & $\begin{array}{l}\text { ASIA Exam } \\
\text { Grade }\end{array}$ \\
\hline $\mathrm{C} 2-\mathrm{C} 3$ and $\mathrm{T} 1$ fracture & Vertebral artery & Heparin drip & Aspirin & C2-C3 ACDF & $\mathrm{C}=>\mathrm{D}$ \\
\hline $\mathrm{C} 3-\mathrm{C} 4$ fracture & Vertebral artery & Heparin drip & $\begin{array}{l}\text { Heparin drip }=>\text { aspirin } \\
\text { and clopidogrel }\end{array}$ & C3-C4 ACDF & $\mathrm{D}=>\mathrm{E}$ \\
\hline $\mathrm{C} 4-\mathrm{C} 5$ pedicle fracture & Vertebral artery & Heparin drip & Heparin drip $=>$ aspirin & C5-C6 ACDF & $\mathrm{E}$ \\
\hline C5-C6 fracture & Vertebral artery & Heparin drip & Heparin drip $=>$ aspirin & C5-C6 ACDF & $\mathrm{E}$ \\
\hline C5 lamina fracture & Vertebral artery & Heparin drip & Heparin drip & C5-C6 ACDF & $\mathrm{E}$ \\
\hline C5 fracture & Vertebral artery & Heparin drip & Aspirin & C5-C6 ACDF & $\mathrm{E}$ \\
\hline C6-T1 fracture & Vertebral artery & Heparin drip & $\begin{array}{l}\text { Heparin drip }=>\text { aspirin } \\
\text { and clopidogrel }\end{array}$ & C5-C6 ACDF & $\mathrm{D}$ \\
\hline C5-C6 dislocation & Vertebral artery & Heparin drip & Heparin drip $=>$ aspirin & C5-C6 ACDF & E \\
\hline C5-C7 lamina fracture & $\begin{array}{l}\text { Internal carotid } \\
\text { artery }\end{array}$ & Heparin drip & $\begin{array}{l}\text { Heparin drip }=>\text { aspirin } \\
\text { and clopidogrel }\end{array}$ & C5-C7 ACDF & $\mathrm{E}$ \\
\hline C6-C7 fracture dislocation & Vertebral artery & Heparin drip & Heparin drip $=>$ aspirin & C6-C7 ACDF & B \\
\hline C5-C6 fracture & $\begin{array}{l}\text { Internal carotid } \\
\text { artery }\end{array}$ & $\begin{array}{l}\text { Heparin drip, aspirin, } \\
\text { and clopidogrel }\end{array}$ & Clopidogrel & C4-C7 corpectomy & A \\
\hline Occiput-C2 fracture & $\begin{array}{l}\text { Internal carotid } \\
\text { artery }\end{array}$ & $\begin{array}{l}\text { Heparin drip, aspirin, } \\
\text { and clopidogrel }\end{array}$ & Clopidogrel & Occipito-C2 PSF & A \\
\hline $\mathrm{C} 2$ fracture & Vertebral artery & Heparin drip & Heparin drip $=>$ aspirin & C1-C4 PSF & $\mathrm{E}$ \\
\hline $\begin{array}{l}\mathrm{C} 3-\mathrm{C} 4 \text { and } \mathrm{C} 6-\mathrm{C} 7 \text { and } \\
\text { T11 fracture }\end{array}$ & $\begin{array}{l}\text { Internal carotid } \\
\text { artery }\end{array}$ & Heparin drip & $\begin{array}{l}\text { Heparin drip }=>\text { aspirin } \\
\text { and clopidogrel }\end{array}$ & $\begin{array}{l}\mathrm{C} 2-\mathrm{T} 2 \text { and } \\
\mathrm{T} 10-\mathrm{T} 12 \mathrm{PSF}\end{array}$ & $\mathrm{E}$ \\
\hline
\end{tabular}

achieved. No patient had a drain in longer than 48 hours. For patients who were receiving anticoagulants, appropriate laboratory values were monitored (serial PTT and complete blood cell count). Anticoagulation therapy and outpatient management were overseen by the vascular service at our trauma center. All patients were under observation in the intensive care unit for a minimum of 48 hours postoperatively. Serial neurologic examinations were performed every hour for the first 24 to 48 hours. All patients were followed until complete fusion was obtained and confirmed by radiographic imaging. Time to complete fusion ranged from 12 weeks to 6 months.

\section{Statistical Analysis}

Patient evaluations included an ASIA form that was completed before and after surgery, recording any changes in neurologic function. By applying numeric scores to these ASIA grades, injury severity was compared between the patients who received anticoagulants and those who did not. A grade A injury was assigned a score of 1 , grade $\mathrm{B}$ was assigned a score of 2, C was assigned 3, D was assigned 4, and a grade E injury was given a score of 5. A 2-tailed Student $t$ test was employed to detect differences. All statistics were determined using SPSS software version 22 (IBM Corporation, Chicago, IL). Statistical significance was accepted at $P<.05$.

\section{RESULTS}

Of the 63 patients with blunt cervical spine trauma requiring stabilization, 14 had an associated cerebrovascular injury requiring anticoagulation treatment $(22 \%)$. There were 10 vertebral artery and 4 internal carotid artery injuries in these patients. The vascular injury did not influence the type of stabilization procedure performed. A total of 11 of these 14 patients underwent anterior cervical procedures, and 3 had posterior fixation (Table 1).

The cerebrovascular injury cohort recorded 2 complications and 1 incident of supratherapeutic PTT. The 2 complications included a case of postoperative pneumonia and a case of anterior fixation failure requiring revision surgery. Neither of the complications was associated with anticoagulant therapy, and no complication came from the elevated PTT incident. ASIA grades did not decline in any of these 14 patients, with 2 patients actually demonstrating improvement (Table 1). No bleeding events, thrombosis, surgical site infections, or other complications related to anticoagulation treatment were noted.

The pneumonia occurred in the immediate postoperative period. The patient required intubation and prolonged intensive care treatment lasting 2 weeks. The patient had a C5 facet fracture with a C5 disc disruption, and the vascular injury was of the right vertebral artery. This patient underwent a C5 to C6 anterior cervical decompression and 
Table 2. Vertebral injury sustained, stabilization received, and American Spinal Injury Association (ASIA) exam grade for each participant in our control cohort who underwent fixation without perioperative anticoagulation therapy.

\begin{tabular}{|c|c|c|c|c|c|}
\hline Vertebral Injury & $\begin{array}{c}\text { Surgical } \\
\text { Procedure }\end{array}$ & $\begin{array}{l}\text { ASIA Exam } \\
\text { Grade }\end{array}$ & Vertebral Injury & $\begin{array}{c}\text { Surgical } \\
\text { Procedure }\end{array}$ & $\begin{array}{l}\text { ASIA Exam } \\
\text { Grade } \\
\end{array}$ \\
\hline C2-C3 disc disruption & C2-C3 ACDF & $\mathrm{E}$ & C3 epidural abscess & C3 corpectomy & $\mathrm{C}=>\mathrm{E}$ \\
\hline C3-C4 stenosis & C3-C4 ACDF & $\mathrm{D}=>\mathrm{E}$ & $\mathrm{C} 3-\mathrm{C} 4$ fracture dislocation & C3-C7 corpectomy & $\mathrm{C}$ \\
\hline C3-C4 disc disruption & C3-C4 ACDF & A & $\begin{array}{l}\mathrm{C} 3-\mathrm{C} 4 \text { and } \mathrm{C} 4-\mathrm{C} 5 \text { and } \mathrm{C} 5-\mathrm{C} 6 \text { disc } \\
\text { disruption }\end{array}$ & C3-C6 corpectomy & $\mathrm{D}$ \\
\hline C3-C4 disc disruption & $\mathrm{C} 3-\mathrm{C} 4 \mathrm{ACDF}$ & $\mathrm{D}$ & C5-C6 myelopathy and cord injury & C5 corpectomy & E \\
\hline $\mathrm{C} 3-\mathrm{C} 4$ disc disruption & C3-C4 ACDF & $\mathrm{E}$ & $\mathrm{C} 4-\mathrm{C} 5$ and $\mathrm{C} 5-\mathrm{C} 6$ disc disruption & C5 corpectomy & E \\
\hline $\mathrm{C} 3-\mathrm{C} 4$ and $\mathrm{C} 4-\mathrm{C} 5$ disc disruption & C3-C5 ACDF & E & $\mathrm{C} 5-\mathrm{C} 6$ and $\mathrm{C} 6-\mathrm{C} 7$ disc disruption & C5 corpectomy & E \\
\hline C4-C5 myelopathy & C4-C5 ACDF & E & C5 cord injury & C5 corpectomy & $\mathrm{D}=>\mathrm{E}$ \\
\hline $\mathrm{C} 4-\mathrm{C} 5$ and $\mathrm{C} 5-\mathrm{C} 6$ disc disruption & C4-C6 ACDF & E & $\mathrm{C} 4-\mathrm{C} 5$ and $\mathrm{C} 5-\mathrm{C} 6$ disc disruption & C5 corpectomy & $\mathrm{D}$ \\
\hline C4-C5 disc disruption & C4-C6 ACDF & E & C4-C5 facet fracture & C4-C5 PSF & $\mathrm{E}$ \\
\hline C5-C6 myelopathy & C5-C6 ACDF & $\mathrm{D}=>\mathrm{E}$ & C6-T1 posterior fracture & C6-T2 PSF & E \\
\hline C5-C6 myelopathy & C5-C6 ACDF & E & $\mathrm{C} 3-\mathrm{T} 1$ fracture & C3-T2 PSF & A \\
\hline C5-C6 disc disruption & C5-C6 ACDF & E & C6 fracture dislocation & PSF C3-T2 & $\mathrm{E}$ \\
\hline C5-C6 disc disruption & C5-C6 ACDF & $\mathrm{E}$ & C3-C5 fracture & C3-C7 PSF & $\mathrm{E}$ \\
\hline C5-C6 fracture dislocation & C5-C6 ACDF & $\mathrm{E}$ & C3-C4 fracture dislocation & C3-C4 PSF & A \\
\hline C5-C6 fracture dislocation & C5-C6 ACDF & $\mathrm{D}$ & C6-C7 dislocation & C6-C7 PSF & $\mathrm{E}$ \\
\hline C5-C6 fracture dislocation & C5-C6 ACDF & A & C6-C7 fracture dislocation & C6-C7 PSF & A \\
\hline C6-C7 fracture dislocation & C6-C7 ACDF & $\mathrm{E}$ & C7-T1 fracture & C5-T3 PSF & E \\
\hline C6-C7 facet fracture & C6-C7 ACDF & E & C4-C5 facet fracture & C4-C5 PSF & $\mathrm{D}$ \\
\hline C6-C7 facet fracture & C6-C7 ACDF & E & C6-C7 fracture dislocation & C3-T1 PSF & $\mathrm{D}$ \\
\hline C6-C7 disc disruption & C6-C7 ACDF & E & C6-C7 fracture & C2-C6 PSF & $\mathrm{E}$ \\
\hline C6-C7 disc disruption & C6-C7 ACDF & E & C4-C5 fracture dislocation & C4-C5 PSF & E \\
\hline C6-C7 disc disruption & C6-C7 ACDF & E & C5-C6 fracture & C4-T1 PSF & $\mathrm{E}$ \\
\hline C6-C7 disc disruption & C6-C7 ACDF & E & C6-C7 dislocation & C6-C7 PSF & B \\
\hline C6-C7 disc disruption & C6-C7 ACDF & $\mathrm{D}$ & C4-C5 fracture dislocation & C3-C6 PSF & B \\
\hline C3-C4 and C4-C5 myelopathy & $\begin{array}{l}\mathrm{C} 3-\mathrm{C} 4 \mathrm{ACDF} \text { and } \\
\mathrm{C} 5 \text { corpectomy }\end{array}$ & $\mathrm{D}=>\mathrm{E}$ & & & \\
\hline
\end{tabular}

Abbreviations: ACDF, anterior cervical discectomy and fusion; PSF, posterior spinal fusion.

fusion. Respiratory distress and reintubation occurred within the first 24 hours postoperatively, and bronchoscopic lavage was positive for bacterial pneumonia. The patient responded to antibiotic treatment and had no adverse effects.

The anterior fixation failure was identified at the 4-week mark on follow-up radiographs, associated with continued pain. This patient had a C6 to C7 fracture dislocation with a right vertebral artery injury. Initially, the patient had a Gardner Wells Tongs application and closed reduction followed by a C6 to $\mathrm{C} 7$ anterior cervical decompression and fusion. At the 4-week clinic visit, the patient did not present with neurologic changes on examination and was taken back to the operating room for a $\mathrm{C} 6$ to $\mathrm{T} 1$ posterior stabilization. The patient was on acetylsalicylic acid for the vascular injury at the time of the revision procedure, which was held for 48 hours postoperatively. The patient's fracture healed without further difficulties. Neurologic status did not change in the perioperative period.

One incident of postoperative supratherapeutic PTT was documented. It occurred 8 hours after a C5 to C6 anterior cervical decompression and fusion for a $\mathrm{C} 5$ facet fracture. The patient had a PTT value of 90, and the heparin drip was held until the PTT returned to the therapeutic range of 40 to 60 seconds. The patient suffered no adverse effects.

In comparison, 49 patients were identified for a control group. These patients had no identifiable cerebrovascular injury and were at no time receiving anticoagulation therapy. Anterior stabilization was performed in 33 patients and posterior stabilization in 16 patients (Table 2). Five complications were noted in this cohort. The complications included 3 cases of postoperative bacterial pneumonia requiring reintubation and antibiotic treatment (1 resulting in a mortality), 1 case of acute postoperative respiratory failure (resulting in a mortality), and 1 case of anterior fixation failure requiring revision surgery.

The anterior fixation failure sustained a C6 to C7 disc injury with posterior longitudinal ligament disruption and cervical instability. A C6 to C7 anterior cervical decompression and fusion was performed that resulted in a fixation failure. The patient was converted to a posterior spinal fusion with a good outcome. ASIA grades revealed no deterioration, with 5 patients exhibiting improvement (Table 2). 
Using the numeric ASIA grades, the patients who were receiving anticoagulation treatment and those who were not had similar postoperative mean scores of 3.93 versus 4.16, respectively. The difference between the two was not significantly different $(P=.57)$.

\section{DISCUSSION}

Controversy exists concerning the necessity of anticoagulation treatment following blunt traumatic vertebral artery injuries and their associated bleeding risks in those requiring spinal surgery. ${ }^{8}$ Studies have shown better neurologic outcomes in patients who received anticoagulation treatment for cerebrovascular injury before symptoms arise. ${ }^{1,8}$ Treating patients early with an anticoagulant has been shown to prevent progression to stroke or ischemia in patients with cerebrovascular (vertebral or carotid artery) injury. ${ }^{1-3}$ Miller et $\mathrm{al}^{2}$ found that more than $50 \%$ of untreated carotid and vertebral artery injuries progressed to stroke, whereas with early anticoagulation treatment, less than $6 \%$ made this progression. These data encourage early anticoagulation therapy despite a necessary spinal decompression and fusion operation.

Current orthopedic literature does not provide sufficient recommendations regarding safety and timing of stabilization operations for cervical spine trauma patients who require anticoagulation secondary to an associated blunt cerebrovascular injury. Several articles describe the occurrence of epidural hematomas in a nonoperative setting as a result of anticoagulation treatment, ${ }^{9-11}$ whereas additional papers provide recommendations concerning the initiation of prophylaxis for deep vein thrombosis postoperatively ${ }^{9,12-14}$; however, there is sparse literature concerning safe and acceptable anticoagulation during cervical spine surgery. Likewise, outcomes following this surgical scenario are sparsely reported. A review completed by Cheng et $\mathrm{al}^{16}$ conveyed the risk of major bleeding and hematoma in these situations to be $0 \%$ to $4.3 \%$, depending on the type of anticoagulant used. Although holding anticoagulants until after surgery is ideal to avoid potential complications, this cannot always be done.

Despite these concerns, this retrospective review did not reveal any complications related to anticoagulation treatment for cerebrovascular injury in patients undergoing cervical spine stabilization. As with all spine stabilization procedures, this patient population requires close postoperative management with drain placement, frequent neurologic examinations, and regular monitoring of laboratory values while in an intensive care setting. As a retrospective analysis, this study has innate limitations concerning the accuracy and completeness of injury, procedure, and complication coding. In addition, the small number of patients identified who underwent spinal surgery while being treated with anticoagulation therapy leaves our evaluation and comparison underpowered. Although the sample size was small, the results of this study suggest that in emergent settings patients who require anticoagulation treatment for a cerebrovascular injury can undergo successful cervical spine decompression and fusion procedures. Before this study can be generalized to a wider population of patients, studies involving a greater number of patients with more diverse spinal stabilization procedures will be required.

\section{REFERENCES}

1. Biffl WL, Moore EE, Elliott JP, et al. The devastating potential of blunt vertebral arterial injuries. Ann Surg. 2000;231(5):672-681.

2. Miller PR, Fabian TC, Bee TK, et al. Blunt cerebrovascular injuries: diagnosis and treatment. $J$ Trauma. 2001;51(2):279-281.

3. Mueller CA, Peters I, Podlogar M, et al. Vertebral artery injuries following cervical spine trauma: a prospective observational study. Eur Spine J. 2011;20(12):2202-2209. doi: 10.1007/ s00586-011-1887-2.

4. Biffl WL, Moore EE, Ryu RK, et al. The unrecognized epidemic of blunt carotid arterial injuries: early diagnosis improves neurologic outcome. Ann Surg. 1998;228(4):462-470.

5. Berne JD, Cook A, Rowe SA, Norwood SH. A multivariate logistic regression analysis of risk factors for blunt cerebrovascular injury. J Vasc Surg. 2010;51(1):57-64. doi: 10. 1016/j.jvs.2009.08.071

6. Bromberg WJ, Collier BC, Bee TK, et al. Blunt cerebrovascular injury practice management guidelines: the Eastern Association for the Surgery of Trauma. J Trauma Acute Care Surg. 2010;68(2):471-477. doi: 10.1097/TA. 0b013e3181cb43da.

7. Cothren CC, Biffl WL, Moore EE, Kashuk JL, Johnson JL. Treatment for blunt cerebrovascular injuries: equivalence of anticoagulation and antiplatelet agents. Arch Surg. 2009;144(7):685-690. doi: 10.1001/archsurg.2009.111.

8. Esnault P, Cardinale M, Boret H, D'Aranda E, et al. Blunt cerebrovascular injuries in traumatic brain injury: incidence, risk factors, and evolution. $J$ Neurosurg. 2016;127(1):1-7. doi: 10.3171/2016.4.JNS152600.

9. Tawk C, El Hajj Moussa M, Zgheib R, Nohra G. Spontaneous epidural hematoma of the spine associated with 
oral anticoagulants: 3 case studies. Int J Surg Case Rep. 2015;13:8-11. doi: 10.1016/j.ijscr.2015.05.022.

10. Kirazli Y, Akkoc Y, Kanyilmaz S. Spinal epidural hematoma associated with oral anticoagulation therapy. Am J Phys Med Rehabil. 2004;83(3):220-223.

11. Phuong LK, Wijdicks EF, Sanan A. Spinal epidural hematoma and high thromboembolic risk: between Scylla and Charybdis. Mayo Clin Proc. 1999;74(2):147-149. doi: 10.4065/ 74.2.147.

12. Al-Dujaili TM, Majer CN, Madhoun TE, Kassis SZ, Saleh AA. Deep venous thrombosis in spine surgery patients: incidence and hematoma formation. Int Surg. 2012;97(2):150154. doi: 10.0738/CC71.1.

13. Bryson DJ, Uzoigwe CE, Braybrooke J. Thromboprophylaxis in spinal surgery: a survey. J Orthop Surg Res. 2012;7(1):14. doi: 10.1186/1749-799X-7-14.

14. Fang MC, Maselli J, Lurie JD, Lindenauer PK, Pekow PS, Auerbach AD. Use and outcomes of venous thromboembolism prophylaxis after spinal fusion surgery. J Thromb Haemost. 2011;9(7):1318-1325. doi: 10.1111/j.1538-7836.2011. 04326.x.

15. Steib A, Hadjiat F, Skibba W, Steib JP; French Spine Surgery Society. Focus on perioperative management of anticoagulants and antiplatelet agents in spine surgery. Orthop
Traumatol: Surg Res. 2011;975:S102-S106. doi: 10.1016/j.otsr. 2011.07.005.

16. Cheng JS, Arnold PM, Anderson PA, Fischer D, Dettori JR. Anticoagulation risk in spine surgery. Spine. 2010;35(9S):S117-S124. doi: 10.1097/BRS.0b013e3181d833d4.

Disclosures and COI: The authors received no funding for this study and report no conflicts of interest.

Corresponding Author: Sean M. Mitchell, MD, University of Arizona Orthopedic Residency, 1320 North 10th St, Ste A, Phoenix, AZ 85006. Phone: (630) 696-2195; Email: smmitche5@gmail. com.

Published 29 February 2020

This manuscript is generously published free of charge by ISASS, the International Society for the Advancement of Spine Surgery. Copyright (c) 2020 ISASS. To see more or order reprints or permissions, see http://ijssurgery.com. 